\title{
Induced-Charge Enhancement of Diffusion Potential in Membranes with Polarizable Nanopores
}

\author{
I.I. Ryzhkov*, D.V. Lebedev, V.S. Solodovnichenko, A.V. Shiverskiy, M.M. Simunin \\ Institute of Computational Modelling $S B R A S$, \\ Federal Research Center KSC SB RAS, Akademgorodok 50, Krasnoyarsk, Russia
}

\begin{abstract}
We report a significant enhancement of diffusion potential in uncharged membranes with polarizable nanopores in comparison with uncharged dielectric membranes. The electric field generated by diffusion of ions with different mobilities induces non-uniform surface charge. It results in the Donnan potentials, which contribute to the total membrane potential. The calculations on the basis of modified Space-Charge model are in good agreement with experiments in $\mathrm{KCl}$ and $\mathrm{NaCl}$ solutions. The enhanced sensitivity of membrane potential to ion mobilities ratio, electrolyte concentration, and pore size can be advantages for applications in electrochemical sensing and design of nanofluidic devices.
\end{abstract}

The understanding of ion transport in nanopores and nanochannels is of fundamental importance in various areas of science and technology, such as separation and purification [1], energy conversion [2], chemical sensors [3], and cell physiology [4]. Nowadays, a lot of research is focused on the development of smart nanopores and nanochannels with tunable ion transport. The latter is realized by combining the pore design strategy (geometry and surface chemical modification) with external stimuli, such as transmembrane potential, solution $\mathrm{pH}$, temperature, light, etc. [5]. The ion transport through charged/uncharged membranes separating electrolytes with different concentrations has received much attention of researchers in a recent decade in various contexts including the design of nanofluidic devices [6], power generation from salinity gradients [7], potentiometric sensing [8], asymmetric diffusion transport [9], and osmotic flow generation [10].

A new class of membranes containing gold nanotubules that span a complete thickness of a porous polymeric support was suggested in [11]. It was shown that their selectivity can be reversible switched from anion to cation by changing the potential applied to the conductive membrane surface. Note that when charged species are transported through a nanopore with polarizable conductive walls, they can induce a surface charge, and thus alter the pore transport characteristics. The induced-charge electrokinetic phenomena are actively investigated nowadays [12] due to potential applications in microfluidic pumping and mixing [13], particle manipulation [14], capacitive deionization [15], and control of ionic transport in nanochannels [16].

The ionic permselectivity of a nanoporous membrane can be assessed by measuring the potential difference at zero current, which appears between two electrolyte solutions with different concentrations separated by the membrane $[17,18]$. The membrane potential is usually represented as a sum of Donnan potentials arising from equilibrium between diffusion and electric forces at the membrane/solution interface, and diffusion potential associated with differ- ent mobilities of ions diffusing along the concentration gradient [19]. The contribution of Donnan potentials is dominant for highly charged membranes, while the contribution of diffusion potential can be significant for moderately charged membranes at large concentration gradients and/or ion mobilities ratio. For an uncharged membrane in 1-1 electrolyte solution, the diffusion potential is given by [20]

$$
\Delta \Phi=\frac{R_{g} T}{F} \frac{D-1}{D+1} \ln \frac{C_{L}}{C_{R}},
$$

where $R_{g}$ is the ideal gas constant, $T$ is the temperature, $F$ is the Faraday constant, $C_{L}$ and $C_{R}$ are the electrolyte concentrations, and $D=D_{+} / D_{-}$is the ratio of ion diffusion coefficients (or mobilities). The diffusion potential is zero when $D=1$, while it coincides with the Nernst potential when $D \rightarrow 0$ or $\infty$.

In this Letter, we show both theoretically and experimentally that the diffusion potential of an uncharged nanopore with polarizable conductive walls can be greatly enhanced in comparison with nonpolarizable case described by Eq. (1). It occurs due to induction of surface charge in the electric field generated by diffusion of ions with different mobilities.

Theoretical model. A membrane is modelled as an array of cylindrical pores of length $L$ and radius $R$, which connect two reservoirs with 1-1 electrolyte of concentrations $C_{L}$ and $C_{R}$. The flow and ion transport are described by the Space-Charge model derived from Navier-Stokes, Nernst-Planck, and Poisson equations [21]. We introduce characteristic scales of radial $R$ and axial $L$ lengths, concentration $C_{0}=1$ $\mathrm{mM}$, electric potential $R_{g} T / F$, ion fluxes $D_{+} C_{0} / L$, velocity $D_{+} / L$, pressure $C_{0} R_{g} T$, and surface charge density $\varepsilon \varepsilon_{0} R_{g} T / F R$. Here $\varepsilon \varepsilon_{0}$ is the dielectric constant. The dimensionless potential $\varphi$, ion concentrations $c_{ \pm}$, and pressure $p$ are written as [21]

$$
\begin{gathered}
\varphi=\phi_{v}(x)+\psi(r, x), \quad c_{ \pm}=c_{v}(x) \exp (\mp \psi(r, x)), \\
p=p_{v}(x)+2 c_{v}(x) \cosh (\psi(r, x)) .
\end{gathered}
$$

The function $\psi$ satisfies the Poisson equation

$$
\frac{1}{r} \frac{\partial}{\partial r}\left(r \frac{\partial \psi}{\partial r}\right)=\frac{c_{v}}{\lambda^{2}} \sinh \psi,
$$


with boundary conditions $\partial \psi / \partial r(0, x)=0$ and

$$
\begin{gathered}
\psi(1, x)=\varphi_{s}-\phi_{v}(x) \quad(\text { const. surface potential }) \\
\text { or } \quad \frac{\partial \psi}{\partial r}(1, x)=\sigma_{s} \quad \text { (const. surface charge) }
\end{gathered}
$$

Here $\lambda=\sqrt{\varepsilon \varepsilon_{0} R_{g} T / 2 F^{2} C_{0}} / R$ is the dimensionless Debye length. The virtual variables $\phi_{v}, c_{v}, p_{v}$ are found by solving the ODE system

$$
\left(\frac{d p_{v}}{d x}, \frac{1}{c_{v}} \frac{d c_{v}}{d x}, \frac{d \phi_{v}}{d x}\right)^{T}=L\left(J_{V}, J_{I}, J_{C}\right)^{T},
$$

where $J_{V}$ is the volume flux (velocity), $J_{I}=J_{+}+J_{-}$ is the ion flux, $J_{C}=J_{+}-J_{-}$is the ionic current (all fluxes are dimensionless and cross-sectionally averaged), while $L$ is the symmetric $3 \times 3$ matrix, which coefficients $L_{i j}(x)$ are calculated using the solution $\psi(r, x)$, see Supplementary Material [22]. The membrane potential is measured at zero current $J_{C}=0$ and equal pressures in both reservoirs. In this case, system (5) becomes

$$
\begin{gathered}
\frac{d p_{v}}{d x}=L_{11} J_{V}+L_{12} J_{I}, \quad \frac{1}{c_{v}} \frac{d c_{v}}{d x}=L_{12} J_{V}+L_{22} J_{I} \\
\frac{d \phi_{v}}{d x}=L_{13} J_{V}+L_{23} J_{I}
\end{gathered}
$$

The corresponding boundary conditions are

$$
\begin{aligned}
& x=0: \quad p_{v}=-2 c_{L}, \quad c_{v}=c_{L}, \quad \phi_{v}=0, \\
& x=1: \quad p_{v}=-2 c_{R}, \quad c_{v}=c_{R}, \quad \phi_{v}=\Delta \varphi,
\end{aligned}
$$

where $\Delta \varphi$ is the dimensionless potential difference between the reservoirs (membrane potential).

We assume that the conductive nanopore wall is ideally polarizable and there is no charge exchange between the nanopore wall and electrolyte. The polarization by electric field, which develops inside the pore, causes the redistribution of surface charge. In this case, the floating boundary condition should be used [16]:

$$
\int_{0}^{1} \frac{\partial \psi}{\partial r} d x=\sigma_{t}
$$

Here $\sigma_{t}=\sigma_{T}\left(\varepsilon \varepsilon_{0} R_{g} T / F R\right)^{-1}$ is the dimensionless total surface charge density, while the dimensional total surface charge is given by $\sigma_{T} 2 \pi R L$.

In this Letter, we propose a method of solving the presented model. To the best of our knowledge, this is the first attempt of applying the Space-Charge model to nanopores with polarizable walls. The difficulty lies in solving Eq. (2) with boundary condition (3). The virtual potential $\phi_{v}(x)$ is found by integration of system (6) with coefficients $L_{i j}(x)$, which in turn depend on the solution $\psi(r, x)$ of problem (2), (3). It makes the system strongly coupled and non-linear.

Let us express $d x=d c_{v}\left(c_{v}\left(L_{12} J_{V}+L_{22} J_{I}\right)\right)^{-1}$ from the second equation in (6) and substitute it into the first and third equations. Integration of resulting equations (6) over the pore length with the help of boundary conditions $(7)$ gives

$$
\begin{gathered}
\int_{c_{L}}^{c_{R}} \frac{L_{11} J+L_{12}}{c_{v}\left(L_{12} J+L_{22}\right)} d c_{v}+2\left(c_{R}-c_{L}\right)=0, \\
J_{I}=\int_{c_{L}}^{c_{R}} \frac{d c_{v}}{c_{v}\left(L_{12} J+L_{22}\right)}, \\
\phi_{v}\left(c_{v}\right)=\int_{c_{L}}^{c_{v}} \frac{L_{13} J+L_{23}}{c_{v}\left(L_{12} J+L_{22}\right)} d c_{v},
\end{gathered}
$$

where $J=J_{V} / J_{I}$. It follows from (2), (3), and (11) that one can write $\psi=\psi\left(r, c_{v}\right), \phi_{v}=\phi\left(c_{v}\right), L_{i j}=$ $L_{i j}\left(c_{v}\right)$, see also $[21,22]$.

The calculation is performed as follows. For $a$ non-polarizable dielectric nanopore with constant surface charge density $\sigma_{s}$, problem (2), (4) is solved numerically for a set of successive values $c_{v}=c_{v i}$, $i=0, \ldots, n$, where $c_{v 0}=c_{L}, c_{v n}=c_{R}$. Then the ratio of fluxes $J=J_{V} / J_{I}$ is found numerically from (9), and the ion flux $J_{I}$ is obtained from (10). The potential difference between reservoirs $\Delta \varphi=\phi_{v}\left(c_{R}\right)$ is determined from (11), while the virtual variables are found by integration of (6), (7). For a polarizable conductive nanopore with constant total surface charge density $\sigma_{t}$, the surface potential $\varphi_{s}$ should be determined in order to satisfy the floating boundary condition (8). In this case, problem (2), (3) is solved for each $c_{v i}$ at fixed $\varphi_{s}$ and $J$. Note that $\phi_{v}\left(c_{v 0}\right)=\phi_{v}\left(c_{L}\right)=0$. The value $\phi_{v}\left(c_{v i}\right)$ is found iteratively starting from $\phi_{v}\left(c_{v, i-1}\right)$ and repeating the solution of (2), (3) followed by application of (11). The whole calculation is performed iteratively to find the fluxes ratio $J$ from (9). Then $J_{I}$ is calculated from (10) and virtual variables are obtained by integration of (6), (7). It allows to find $\psi(r, x)=\psi\left(r, c_{v}(x)\right)$ and finally calculate the integral in the left-hand side of (8). The whole procedure is iterated to find the surface potential $\varphi_{s}$, with which Eq. (8) is satisfied. Note that when solving the Poisson equation (2), the value of $\psi$ at $r=0$ should be determined in order to satisfy condition (3). The initial approximation for it is found from analytical solution derived in [23].

For a non-polarizable and uncharged $(\sigma=0)$ nanopore, the dimensional potential, concentrations, and pressure do not depend on $r$, and can be determined analytically [18]:

$$
\begin{gathered}
\Phi=\frac{R_{g} T}{F} \frac{D-1}{D+1} \ln \frac{C_{L}}{C_{ \pm}}, \\
C_{ \pm}=C_{L}+\left(C_{R}-C_{L}\right) \frac{x}{L}, \quad P=0 .
\end{gathered}
$$

Membrane preparation and potential measurement. To validate the predictions of theoretical model experimentally, we have synthesized membranes with dielectric as well as conductive nanoporous structure. The membranes are prepared from Nafen ${ }^{\mathrm{TM}}$ alumina nanofibers supplied by ANF Technologies (Estonia) in the form of blocks. The diameter of a single nanofiber 

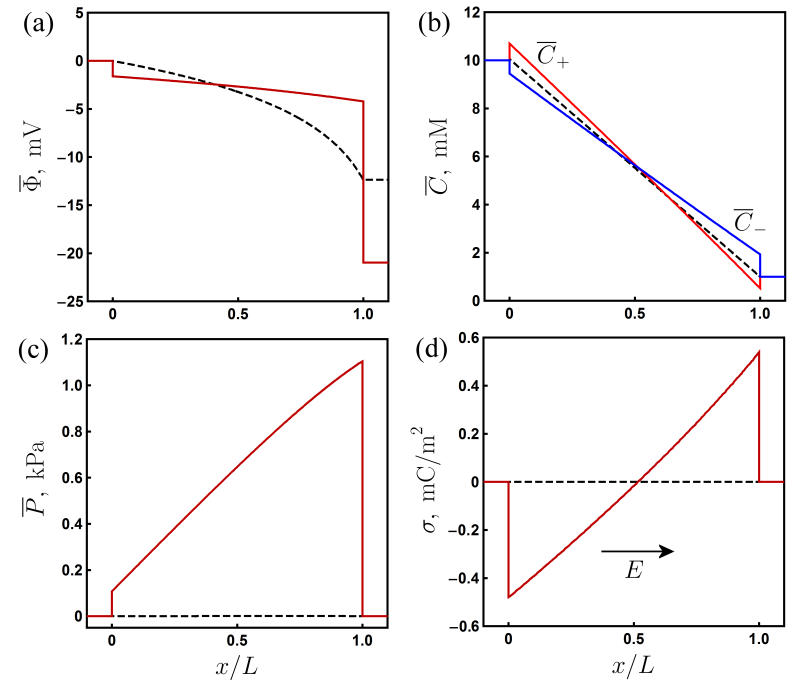

FIG. 1: The cross-sectionally averaged potential (a), concentrations (b), pressure (c), and surface charge density (d) for non-polarizable ( $\sigma=0$, dashed curves) and polarizable $\left(\sigma_{T}=0\right.$, solid curves) nanopores in aqueous $\mathrm{NaCl}$ solution. $R=8 \mathrm{~nm}, L=400 \mu \mathrm{m}, C_{L}=10 \mathrm{mM}, C_{R}=1 \mathrm{mM}$.

is $10-15 \mathrm{~nm}$, and the length is up to $100 \mathrm{~mm}$. The nanofibers are dispersed in deionized water (the weight ratio of Nafen:water is 1:200) and agitated with a magnetic stirrer for 30 minutes followed by 15 minutes of ultrasonic treatment (Sonics \& Materials VC-505, USA). The suspension is filtered through the rough Teflon filter (average pore size of $0.6 \mu \mathrm{m}$ ) and dried in air. The membrane is sintered at $800{ }^{\circ} \mathrm{C}$ during 4 hours to ensure its structural stability in aqueous solutions. The produced membrane is a circular disc with the diameter of $\sim 40 \mathrm{~mm}$ and thickness of $\sim 400 \mu \mathrm{m}$. Chemical vapor deposition (CVD) is used to form conductive carbon layers on the nanofibrous membrane structure. The synthesis is conducted in a homemade reactor at $900{ }^{\circ} \mathrm{C}$ (heating rate of 20 $\left.30{ }^{\circ} \mathrm{C} / \mathrm{min}\right)$ in propane/nitrogen mixture (1/15) with the total flow rate of $4000 \mathrm{~cm}^{3} / \mathrm{min}$ during 60 seconds. The sample is slowly cooled to $150{ }^{\circ} \mathrm{C}$ in the atmosphere of nitrogen. The samples with and without deposited carbon will be referred to as $\mathrm{C}-\mathrm{Nafen}$ membrane and Nafen membrane, respectively. The dielectric Nafen membrane is characterized by the porosity of $75 \%$, specific surface area of $146 \mathrm{~m}^{2} / \mathrm{g}$, and maximum of pore diameter distribution curve at $28 \mathrm{~nm}$. The corresponding parameters of conductive C-Nafen membrane are $62 \%, 107 \mathrm{~m}^{2} / \mathrm{g}$, and $16 \mathrm{~nm}$ [24]. Both types of membranes are hydrophilic.

The membrane potential is measured in $\mathrm{KCl}$ and $\mathrm{NaCl}$ aqueous solutions in a laboratory made electrochemical cell. It consists of two half-cells, between which the membrane is clamped. In each half-cell, reference $4.2 \mathrm{M} \mathrm{Ag} / \mathrm{AgCl}$ electrode is located. Electrodes are connected to the potentsiostat PI-50Pro (Elins, Russia), which measures the cell EMF. First, the solution with fixed concentration $C_{R}$ is placed in both half-cells and kept at room temperature of $25^{\circ} \mathrm{C}$
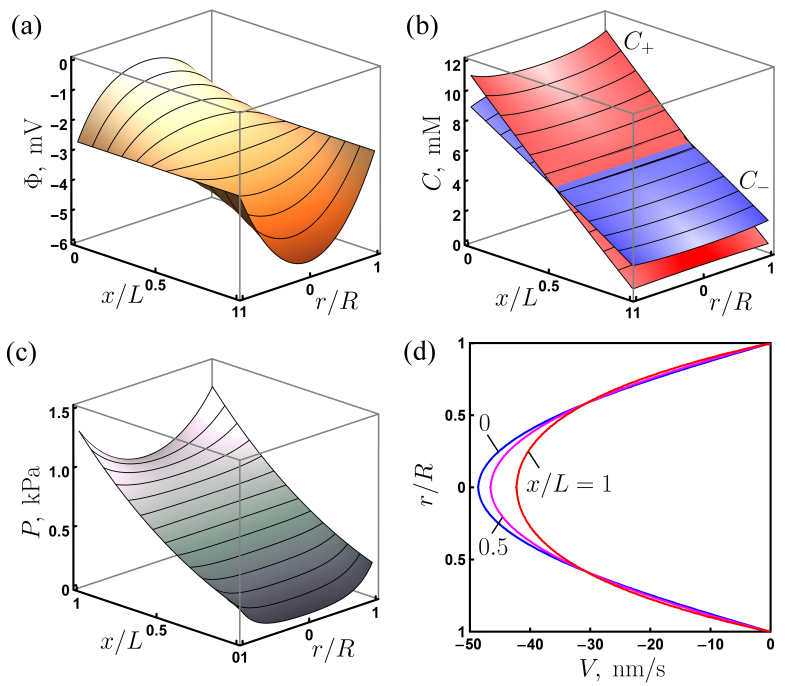

FIG. 2: The potential (a), concentrations (b), pressure (c), and osmotic velocity (d) for a polarizable nanopore with $\sigma_{T}=0$ in aqueous $\mathrm{NaCl}$ solution. $R=8 \mathrm{~nm}, L=400 \mu \mathrm{m}$, $C_{L}=10 \mathrm{mM}, C_{R}=1 \mathrm{mM}$.

during 12 hours. The measurements are performed by successively increasing the electrolyte concentration in the left half-cell. At each step, the system is allowed to equilibrate during $30 \mathrm{~min}$ before the measurement is made. After each series of experiments, the membrane is placed in deionized water for 24 hours to remove the electrolyte solution from the pores. More information about membrane preparation and potential measurements can be found in [24].

Results and discussion. The comparison between non-polarizable and polarizable uncharged nanopores is shown in Fig. 1 for $\mathrm{NaCl}$ aqueous solution with $D_{+}=1.33 \cdot 10^{-9}$ and $D_{-}=2.03 \cdot 10^{-9} \mathrm{~m}^{2} / \mathrm{s}$. In the former case described by Eq. (12), the concentrations of cations and anions coincide. The potential decrease results in the electric field $E=-\nabla \Phi$, which speeds up the slower diffusing cation and retards the faster diffusing anion to make the total ion fluxes equal. In a polarizable pore, this electric field induces the surface charge, which changes almost linearly in the direction from the pore entrance $(x / L=0)$ to the pore exit $(x / L=1)$, while keeping the total surface charge $\sigma_{T}$ zero, see Fig. 1 (d). It results in the higher concentration of cations (anions) at negatively (positively) charged part of the pore, Fig. 1 (b). The separation of charge induces the Donnan potentials at the pore entrance and exit, which both contribute to the enhancement of membrane potential in comparison with non-polarizable pore, Fig. 1 (a). It can be seen from Fig. 2 (a) that the surface potential is constant $(-2.7 \mathrm{mV})$, while the potential increases (decreases) in those regions of pore, where the concentration of cations (anions) is higher. The separation of charge also results in osmotic pressure jumps at the pore entrance and exit, see Figs. 1 (c) and 2 (c). They in turn induce the osmotic flow in the direction of higher 

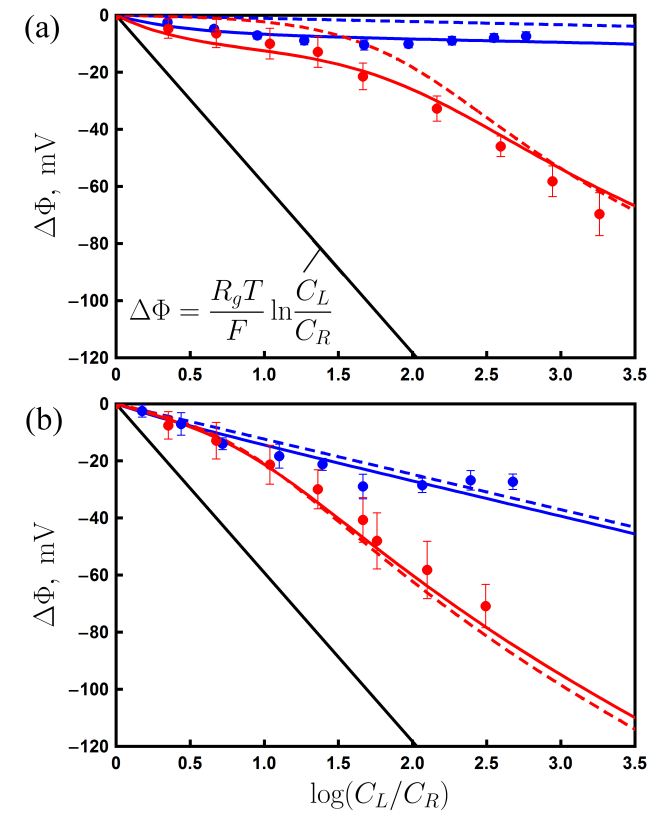

FIG. 3: Membrane potential of Nafen (blue) and C-Nafen (red) membranes in $\mathrm{KCl}$ (a) and $\mathrm{NaCl}$ (b) aqueous solutions. Experimental data (points), calculations for uncharged non-polarizable (blue) and polarizable (red) pores (dashed curves), fitting of experimental data (solid curves), ideal anion selectivity (solid black line). Error bars, 1 s.d.

TABLE I: Experimental cases with fitted values of $\sigma$ (Nafen membranes) or $R$ and $\sigma_{T}$ (C-Nafen membranes).

\begin{tabular}{lcccc}
\hline \hline Electrolyte & \multicolumn{2}{c}{ Aqueous KCl } & \multicolumn{2}{c}{ Aqueous NaCl } \\
Membrane & Nafen & C-Nafen & Nafen & C-Nafen \\
\hline$C_{R}, \mathrm{mM}$ & 1 & 0.1 & 1 & 1 \\
$R, \mathrm{~nm}$ & 14 & 8.8 & 14 & 9.4 \\
$\sigma$ or $\sigma_{T}, \mathrm{mC} / \mathrm{m}^{2}$ & 0.329 & 0.039 & 0.128 & 0.045 \\
\hline \hline
\end{tabular}

salt concentration, Fig. 2 (d). The higher concentration of cations (anions) near the pore entrance (exit) results in the decrease (increase) of velocity in the near-wall region due to the presence of electric field. The opposite situation is observed at the center since the total volume flow rate is constant along the pore.

The calculated membrane potentials of uncharged non-polarizable $(\sigma=0, R=14 \mathrm{~nm})$ and polarizable $\left(\sigma_{T}=0, R=8 \mathrm{~nm}\right)$ membranes in $\mathrm{KCl}$ and $\mathrm{NaCl}$ solutions are shown in Fig. 3 by dashed curves. The induced-charge enhancement of diffusion potential for $\mathrm{KCl}$ solution with almost equal ion diffusion coefficients $\left(D_{+}=1.96 \cdot 10^{-9} \mathrm{~m}^{2} / \mathrm{s}\right.$, $\left.D_{+} / D_{-}=0.96\right)$ is quite significant (more than 16 times at $\log \left(C_{L} / C_{R}\right)=3$ ). For $\mathrm{NaCl}$ solution, the enhancement is around 2.6 times at the same concentration contrast.

The theoretical results are well supported by the experimental data. The measurements for Nafen membrane were performed at $\mathrm{pH}=9.1 \mathrm{in} \mathrm{KCl}$ solution and $\mathrm{pH}=8$ in $\mathrm{NaCl}$ solution, which correspond to the point of zero charge for alumina surface $[25,26]$.
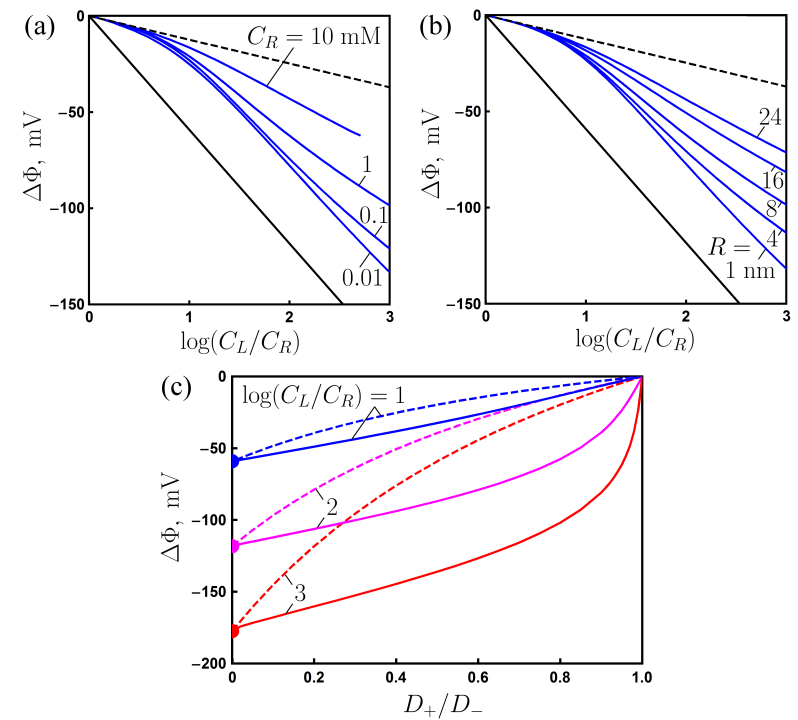

FIG. 4: The effect of concentration $C_{R}$ (a) and pore radius $R$ (b) on the membrane potential in $\mathrm{NaCl}$ aqueous solution for $\sigma_{T}=0$ (blue curves). Membrane potential for $\sigma=0$ (dashed line) and ideal anion selectivity (solid black line). The effect of diffusion coefficients ratio on membrane potential (c) for uncharged non-polarizable (dashed curves) and polarizable (solid curves) nanopores, $R=8 \mathrm{~nm}, C_{R}=0.1$ mM.

The values of surface charge density $\sigma$ obtained by fitting of experimental data to the theoretical model of non-polarizable nanopore with $R=14 \mathrm{~nm}$ are presented in Table I. They are rather low, so the fitted curves only slightly deviate from those corresponding to $\sigma=0$.

For C-Nafen membrane with conductive carbon surface, the adsorption of alkali metal cations on the defects of carbon structure can occur and modify the surface charge $[24,27]$. To minimize this effect, low electrolyte concentrations were used: $C_{R}=0.1 \mathrm{mM}$ for $\mathrm{KCl}$ and $C_{R}=1 \mathrm{mM}$ for $\mathrm{NaCl}$. In this case, the experimental data were fitted to theoretical model of polarizable nanopore to obtain the total surface charge density $\sigma_{T}$ and pore radius $R$, see Table I. The obtained values of $\sigma_{T}$ are positive but rather small, while the $R$ values are in good agreement with low temperature nitrogen adsorption data $(8 \mathrm{~nm})[24]$. So, the fitted curves are close to those of fully uncharged $\left(\sigma_{T}=0\right)$ polarizable membranes in Fig. 3.

The diffusion potential of an uncharged nonpolarizable membrane depends only on the ratio of concentrations and ion diffusion coefficients, see Eq. (1). In the case of membrane with polarizable pores, the situation is strikingly different. Fig. 4 (a) shows that the enhancement of membrane potential becomes stronger with decreasing the concentration $C_{R}$. The calculations reveal that the variation of averaged potential along the pore becomes smaller, while the Donnan potentials at the pore entrance and exit become larger when $C_{R}$ decreases at fixed ratio $C_{L} / C_{R}$, see also Fig. 1. At smaller concentrations, the Debye length is larger, which means a stronger overlap of 
electric double layers created by the induced surface charge. The same effect can be achieved by decreasing the pore radius, see Fig. 4 (b). The membrane potential shows a significant increase when $R$ decreases, but even at large $R$ its enhancement is quite noticeable in comparison with the non-polarizable case.

The effect of ion diffusion coefficients ratio on the membrane potential is shown in Fig. 4 (c). For both non-polarizable and polarizable pores, $\Delta \Phi=0$ when $D=D_{+} / D_{-}=1$, while it approaches the Nernst potential when $D \rightarrow 0$, see also Eq. (1). In the range $0<D<1$, a significant enhancement of diffusion potential in a polarizable pore is observed. It becomes larger with increasing the concentration contrast. Especially unusual is the strong rise of membrane potential magnitude near $D=1$. It means that a very small difference between diffusion coefficients can result in a large change of membrane potential. This conclusion is confirmed by the experimental data in $\mathrm{KCl}$ solution, see Fig. 3 (a). Note that at the same ratio of smaller to larger diffusivity, the magnitude of membrane potential is the same, but its sign is negative when $D_{+}<D_{-}$and positive when $D_{+}>D_{-}$.

To summarize, we have reported a significant enhancement of diffusion potential in polarizable nanoporous membranes in comparison with uncharged dielectric membranes. The Space-Charge model is modified to allow the description of ion transport in a cylindrical pore with constant surface potential. It is shown that the electric field generated by diffusion of ions with different mobilities induces a non-uniform surface charge, which results in charge separation inside the nanopore. The corresponding Donnan potentials appear at the pore entrance and exit leading to the substantial increase of membrane potential in comparison with fully uncharged nanopore. The theoretical findings are confirmed experimentally by measuring the membrane potential in dielectric and conductive nanoporous membranes using $\mathrm{KCl}$ and $\mathrm{NaCl}$ aqueous solutions. The enhancement effect becomes stronger with decreasing the electrolyte concentration and pore radius. A strong sensitivity of membrane potential to the ratio of ion diffusion coefficients is demonstrated. The described phenomenon may find applications in precise determination of ion mobilities, electrochemical sensing, and design of nanofluidic devices for flexible control of ionic transport at the nanoscale.

This work is supported by the Russian Science Foundation, Project 15-19-10017.
[1] H. Strathmann, Ion-Exchange Membrane Separation Processes (Elsevier, Amsterdam, 2004).

[2] A. Cipollina and G. Micale, Sustainable Energy from Salinity Gradients (Elsevier/Woodhead Publishing, 2016).

[3] F.G. Bănică, Chemical Sensors and Biosensors: Fundamentals and Applications (John Wiley \& Sons, Chichester, UK, 2012).

[4] J. Malmivuo and R. Plonsey, Bioelectromagnetism Principles and Applications of Bioelectric and Biomagnetic Fields (Oxford University Press, New York, 1995).

[5] Z.S. Siwy and S. Howorka, Chem. Soc. Rev. 39, 1115 (2010); X. Hou, W. Guo, and L. Jiang, Chem. Soc. Rev., 40, 2385 (2011); M. Tagliazucchi and I. Szleifer, Mater. Today 18, 131 (2015).

[6] W. Sparreboom, A. van den Berg, and J.C.T. Eijkel, Nature nanotech. 4, 713 (2009); C.B. Picallo, S. Gravelle, L. Joly, E. Charlaix, and L. Bocquet, Phys. Rev. Lett. 111, 244501 (2013).

[7] A. Siria, P. Poncharal, A.L. Biance, R. Fulcrand, X. Blase, S.T. Purcell, and L. Bocquer, Nature, 494, 455 (2013).

[8] J. Bobacka, A. Ivaska, and A. Lewenstam, Chem. Rev. 108, 329 (2008).

[9] Z. Siwy, I.D. Kosińska, A. Fuliński, and C.R. Martin, Phys. Rev. Lett. 94, 048102 (2005).

[10] C. Lee, C. Cottin-Bizonne, A.L. Biance, P. Joseph, L. Bocquet, and C. Ybert, Phys. Rev. Lett. 112, 244501 (2014).

[11] M. Nishizawa, V.P. Menon, and C.R. Martin, Science 268, 700 (1995); C.R. Martin, M. Nishizawa, K. Jirage, M. Kang, and S.B. Lee, Adv. Mater. 13, 1351 (2001).
[12] M.Z. Bazant and T.M. Squires, Curr. Op. Coll. Inter. Sci. 15, 203 (2010).

[13] M.Z. Bazant and T.M. Squires, Phys. Rev. Lett. 92, 066101 (2004).

[14] S. Gangwal, O.J. Cayre, M.Z. Bazant, and O.D. Velev, Phys. Rev. Lett. 100, 058302 (2008).

[15] S. Rubin, M.E. Suss, P.M. Biesheuvel, and M. Bercovici, Phys. Rev. Lett. 117, 234502 (2016).

[16] C. Zhao, Y. Song, and C. Yang, Phys. Fluids 27, 012003 (2015).

[17] P. Fievet, B. Aoubiza, A. Szymczyk, and J. Pagetti, J. Membr. Sci. 160, 267 (1999).

[18] A.H. Galama, J.W. Post, H.V.M. Hamelers, V.V. Nikonenko, and P.M. Biesheuvel, J. Membr. Sci. Res. 2, 128 (2016).

[19] Y. Tanaka, Ion Exchange Membranes: Fundamentals and Applications (Elsevier, Amsterdam, 2015).

[20] J.L. Jackson, J. Phys. Chem. 78, 2060 (1974).

[21] P.B. Peters, R. van Roij, M.Z. Bazant, and P.M. Biesheuvel, Phys. Rev. E. 93, 053108 (2016).

[22] See Supplemental Material.

[23] S. Levine, J.R. Marriott, G. Neale, and N. Epstein, J. Coll. Inter. Sci. 52, 136 (1975).

[24] V.S. Solodovnichenko, D.V. Lebedev, V.V. Bykanova, A.V. Shiverskiy, M.M. Simunin, V.A. Parfenov, and I.I. Ryzhkov, Adv. Engineer. Mater. 20, 1700244 (2017).

[25] M. Tschapek, C. Wasowski, and R.M. Torres Sanchez, J. Electroanal. Chem. 74, 167 (1976).

[26] R. Sprycha, J. Coll. Int. Sci. 127, 1 (1989).

[27] G. Mpourmpakis and G. Froudakis, J. Chem. Phys. 125, 204707 (2006). 